\title{
Guideline improved dentists' knowledge but not their clinical decision-making skills
}

\author{
Is a clinical practice guideline on the management of asymptomatic impacted \\ lower third molars effective in changing practice?
}

\begin{abstract}
van der Sanden WJM, Mettes DG, Plasschaert AJM, Grol RPTM, Mulder J, Verdonschot EH. Effectiveness of clinical practice guideline implementation on lower third molar management in improving clinical decision-making: a randomized controlled trial. Eur J Oral Sci 2005; 113:349-354
\end{abstract}

Design This study utilised a two-arm cluster randomized controlled trial, with pre- and post-test assessments.

Intervention General dental practitioners were randomly allocated to intervention and control groups using a random number table. The intervention was multifaceted and consisted of:

- Initial dissemination of a clinical practice guideline (CPG) by mail, and instruction for use of the CPG.

- At 1 month, general information and feedback on 36 simulated patient cases; Second month: individual feedback on 36 simulated patient cases, partially handwritten.

- Third month: interactive meeting with discussion on the CPG.

- Fourth month: reminder (laminated card with overview of CPG recommendations).

- Fifth month: reminder (overview of general recommendations).

- Sixth month: reminder (postcard with overview of CPG recommendations).

- Eleventh month: repeat questionnaire and 36 simulated patient cases.

Outcome measure Outcomes evaluated were the answers given on pre- and postintervention questionnaires and the number of patients in the simulated cases who were referred for removal of their asymptomatic impacted mandibular third molars.

Table 1. Number and percentage of lower third molars referred for removal

\begin{tabular}{|c|c|c|}
\hline Outcome & Test & Control \\
\hline \multicolumn{3}{|l|}{$\begin{array}{l}\text { Total }(n) \text { referred lower third } \\
\text { molars (asymptomatic, sympto- } \\
\text { matic and fully erupted molars) }\end{array}$} \\
\hline At baseline & 461 & 457 \\
\hline At 1 year & 258 & 305 \\
\hline Change (\%) & $\begin{array}{c}44.1(95 \% \mathrm{Cl} \\
39.5-48.6)\end{array}$ & $\begin{array}{c}33.3(95 \% \mathrm{Cl}, \\
28.9-37.6)\end{array}$ \\
\hline Difference (\%) & \multicolumn{2}{|c|}{$10.8(95 \% \mathrm{Cl}, 4.5-17.1)$} \\
\hline Numbers-needed-to-treat & \multirow{2}{*}{\multicolumn{2}{|c|}{10}} \\
\hline $\begin{array}{l}\text { Referred asymptomatic impacted } \\
\text { mandibular third molars }(n)\end{array}$ & & \\
\hline At baseline & 308 & 335 \\
\hline At 1 year & 250 & 299 \\
\hline Change & $19 \%$ decrease & $11 \%$ decrease \\
\hline
\end{tabular}

$\mathrm{Cl}$, Confidence interval.

Address for correspondence: Dr Wil JM van der Sanden, Radboud University Nijmegen Medical Centre, College of Dental Sciences, PO Box 9101, 6500 HB Nijmegen, The Netherlands. E-mail: w.vandersanden@dent.umcn.nl
Results There was a decrease in the number of patients referred for third molar extraction (see Table 1) and also a significant reduction in the mean number of treatment decisions made by the participants in the intervention group that were not in agreement with the CPG (False Positives and False Negatives) (10.5 in the pretest assessment vs 5.7 in the post-test assessment; mean decrease, $4.8 ; 95 \%$ confidence interval, 3.2-6.4). No significant decrease was observed in the control group (mean decrease, $2.4 ; 95 \%$ confidence interval, 0.7-4.2). There was no difference in the proportion of CPG-consistent, true, positive decisions, however.

Conclusions The study demonstrated that the method employed for dissemination and implementation of a CPG on asymptomatic, impacted mandibular third molars improved the knowledge of dentists on this topic, but did not improve their clinical decision-making skills.

\section{Commentary}

The purpose of clinical guidelines is to limit inappropriate variation in clinical practice by outlining evidence-based practice. This paper describes an attempt to improve the implementation of a dental guideline relating to the management of asymptomatic, impacted mandibular third molars.

Although the intervention succeeded in influencing knowledge, as its many facets (feedback, reminders, interactive meeting) were designed to do, it did not influence clinical practice. These results are not just relevant to the management of third molars. This study adds to the large body of evidence demonstrating that increasing knowledge is usually not sufficient to achieve changes in clinical practice, whatever its form. Nevertheless, implementation interventions continue to be designed based on this unsuccessful educational paradigm. A shift is required in how we think about designing interventions to implement guidelines.

Implementation interventions are intended to influence clinicians' behaviour in line with evidence-based practice. It is reasonable to posit that more appropriate design frameworks would be the psychological models specifically created to predict, explain and influence behaviour. These models specify many variables that are more influential in terms of behaviour change than just knowledge; these can be targets for implementation interventions - for example, behavioural attitude, and intention and action plans.

These models have in fact been extensively and successfully applied to patient-related behaviours, but the application of these models to clinical practice is still in its infancy. There is a growing body of literature, however, that supports the use of these models being extended into this area. Perhaps, though, it is not just implementation interventions that need the paradigm shift. We should be examining the design of guidelines themselves, in terms of whether they accord with evidence for presenting recommendations that require behaviour change.

\section{Debbie Bonetti}

Dental Health Services Research Unit, Dundee, Scotland, UK

Evidence-Based Dentistry (2006) 7, 8.

doi:10.1038/sj.ebd.6400386 